\title{
On Dirichlet type problems of polynomial Dirac equations with boundary conditions
}

\author{
Denis Constales * Dennis Grob ${ }^{\dagger} \quad$ Rolf Sören Kraußhar ${ }^{\ddagger}$ \\ Hoai Thu Le ${ }^{\S}$
}

\begin{abstract}
Let $\mathbf{D}_{\mathbf{x}}:=\sum_{i=1}^{n} \frac{\partial}{\partial x_{i}} e_{i}$ be the Euclidean Dirac operator in $\mathbb{R}^{n}$ and let $P(X)=a_{m} X^{m}+\ldots+a_{1} X+a_{0}$ be a polynomial with real coefficients. Differential equations of the form $P\left(\mathbf{D}_{\mathbf{x}}\right) u(\mathbf{x})=0$ are called homogeneous polynomial Dirac equations with real coefficients. In this paper we treat Dirichlet type problems of the a slighly less general form $P\left(\mathbf{D}_{\mathbf{x}}\right) u(\mathbf{x})=f(\mathbf{x})$ (where the roots are exclusively real) with prescribed boundary conditions that avoid blow-ups inside of the domain. We set up analytic representation formulas for the solutions in terms of hypercomplex integral operators and give exact formulas for the integral kernels in the particular cases dealing with spherical and concentric annular domains. The Maxwell and the Klein Gordon equation are included as special subcases in this context.
\end{abstract}

Keywords: polynomial Dirac equations, Dirichlet problems, reproducing kernels, Bergman and Hardy spaces, annular domains, Clifford analysis, Harmonic analysis, Maxwell equations, Helmholtz type equations, Klein-GOrdon equation

MSC Subject-Classification: 30 G 35; 32 A 25, 31 B 20.

*Department of Mathematical Analysis, Ghent University, Building S-22, Galglaan 2, B9000 Ghent, Belgium. Financial support from BOF/GOA 01GA0405 of Ghent University gratefully acknowledged. E-mail: dc@cage.UGent.be

${ }^{\dagger}$ Lehrstuhl A für Mathematik, RWTH Aachen, D-52056 Aachen, Germany. Financial support through a Graduate Fellowship (GFK) from RWTH Aachen University gratefully acknowledged. E-mail: dennis.grob@mathA.rwth-aachen.de

$¥$ Arbeitsgruppe Algebra, Geometrie und Funktionalanalysis, Fachbereich Mathematik, Technische Universität Darmstadt, Schloßgartenstr. 7, D-64289 Darmstadt, Germany. Email: krausshar@mathematik.tu-darmstadt.de

§Institut für Angewandte Analysis, Technische Universität Bergakademie Freiberg, D09596 Freiberg, Germany. Financial support from the Deutscher Akademischer Austausch Dienst (DAAD) via the $\mathrm{PhD} /$ grant A/08/74960 gratefully acknowledged. E-mail: lethu@mailserver.tu-freiberg.de 


\section{Introduction}

Over the last twenty five years the Clifford operator calculus has been proven to be a very efficient toolkit to develop analytic solution representations as well as efficient numerical algorithms for large classes of elliptic and parabolic boundary value problems in higher dimensions. Its range of applications encompasses linear as well as highly non-linear boundary value problems, arising in all areas of physics and engineering such as for instance in electromagnetism, optics, elasticity, fluid dynamics up to modern quantum field theory. The basic theory can be found in summarized form for instance in the books $[16,17,20]$ and elsewhere. Its particular applications to concrete problems from electromagnetism are for instance treated in detail by A. McIntosh and M. Mitrea in [24, 26], by V. V. Kravchenko and M. Shapiro and their collaborators, cf. e.g. [19, 22, 23], by W. Sprößig in [31] and in many other pieces of literature. In this paper we focus ourselves particularly on the treatment of higher dimensional polynomial Dirac equations of arbitrary polynomial degree where the polynomial however possesses real roots only. These encompass homogeneous and inhomogeneous time-harmonic Maxwell equations and Helmholtz type equations with real parameter, more precisely the Klein-Gordon equation, as particular interesting subcases.

To start we briefly recall the well-known fact that in the monochromatic case the electric and magnetic field components in an isotropic medium $\mathcal{D} \subset \mathbb{R}^{3}$ with no charges and currents in its inside are governed by the following set Maxwell equations

$$
\begin{aligned}
\operatorname{rot} \mathbf{H}=\sigma \mathbf{E} & \operatorname{rot} \mathbf{E}=i \omega \mu \mathbf{H} \\
\operatorname{div} \mathbf{H}=0 & \operatorname{div} \mathbf{E}=0 .
\end{aligned}
$$

Here $\sigma:=\sigma^{*}-i \omega \varepsilon$ is the complex electrical conductivity. $\varepsilon$ is the dielectric constant; $\mu$ is the magnetic permeability, $\omega$ the angular frequency, and $\sigma^{*}$ is the medium electrical conductivity which is the reciprocal value of the electrical resistivity $\rho=\frac{1}{\sigma^{*}}$. The value $\sigma$ is the reciprocal value of the electrical impedance. Its imaginary part is exactly zero if and only if the current and voltage are in phase. Then the circuit is purely resistive. In the other cases it is either inductive $(\Im(\sigma)>0)$ or capacitive $(\Im(\sigma)<0)$.

As is further well-known, in the time-harmonic case, the electrical and magnetic fields $\mathbf{E}$ and $\mathbf{H}$ then obey the homogeneous Helmholtz equations

$$
\begin{aligned}
\Delta \mathbf{E}-\Lambda \mathbf{E} & =0 \\
\Delta \mathbf{H}-\Lambda \mathbf{H} & =0
\end{aligned}
$$

where $\Lambda:=-i \omega \mu \sigma^{*}-\omega^{2} \mu \varepsilon=-i \omega \mu \sigma \in \mathbb{C}$. Notice that as soon as the monochromatic case is solved, one can solve the general time-dependent case for instance by the standard Fourier method, also known as time discretization in engineering. 
It should be noticed that from the quantum mechanical point of view this system has another particular meaning in the case where $\Lambda$ is a positive real number. By making the identification $\Lambda=\frac{m^{2} c^{2}}{\hbar^{2}}$ where $m$ is the mass of a particle, $c$ the speed of light and $\hbar$ the Planck number, the solutions to the previous equations can be interpreted as the solutions to the Klein-Gordon equation, see also [21]. These are exactly the stationary solutions to the Schrödinger equation.

The use of Clifford algebra calculus makes it possible to express both systems of second order partial differential equations in terms of first order elliptic partial differential operators in an elegant way, viz the factorization

$$
\begin{aligned}
& (\mathbf{D}-\sqrt{\Lambda})(\mathbf{D}+\sqrt{\Lambda}) \mathbf{E}=0 \\
& (\mathbf{D}-\sqrt{\Lambda})(\mathbf{D}+\sqrt{\Lambda}) \mathbf{H}=0
\end{aligned}
$$

where we choose the branch of $\lambda:=\sqrt{\Lambda}$ such that $\Re(\lambda)>0$. In the special framework of electromagnetism, the number $\lambda$ is physically interpreted as a medium wave number. Here $\mathbf{D}:=\sum_{i=1}^{3} e_{i} \frac{\partial}{\partial x_{i}}$ is the three dimensional Dirac operator factorizing the Euclidean Laplacian viz $\mathbf{D}^{2}=-\Delta$, when the multiplication is understood as Clifford multiplication in the real Clifford algebra $C l_{3}(\mathbb{R})$ which is defined by $e_{i}^{2}=-1$ for $i=1,2,3$ and $e_{i} e_{j}=-e_{j} e_{i}$ for $i \neq j$.

All these particular problems thus appear as very particular examples of general polynomial Dirac equations with real coefficients of the form $P\left(\mathbf{D}_{\mathbf{x}}\right) u(\mathbf{x})=f(\mathbf{x})$ where $P(X)=a_{m} X^{m}+\ldots+a_{1} X+a_{0}$ is a polynomial with real coefficients $a_{0}, \ldots, a_{m}$. Since every complex polynomial splits into linear factors, the general problem is reduced to consider polynomial Dirac equations of the form $\left(\mathbf{D}_{\mathbf{x}}-\right.$ $\left.\lambda_{1}\right) \cdots\left(\mathbf{D}_{\mathbf{x}}-\lambda_{m}\right) u(\mathbf{x})=f(\mathbf{x})$ with complex values $\lambda_{1}, \ldots, \lambda_{m}$ which may appear in multiple form of some of them may be zero. Due to technical reasons we restrict ourselves in this paper to the special situation where the roots $\lambda_{1}, \ldots, \lambda_{m}$ are all elements of out off $\mathbb{R}$ only.

In the particular case $m=1$ and $f \equiv 0$ one deals with the solutions to the homogeneous time-harmonic Maxwell equations. The case dealing with a nonzero function $f$ on the right-hand side treats the case where we have netto charges and currents inside the domain in which we consider the time-harmonic electromagnetic problem. In the case $m=2$ and $\lambda_{2}=\lambda_{1}$ we are dealing with the generalized Helmholtz type equation with parameter $\lambda=\mu \omega \sigma$ where $\sigma$ is the electrical conductivity. The aim of this paper is to set up explicit representation formulas for general Dirichlet type problems of more general inhomogeneous polynomial Dirac equations (with the only restriction that the polynomials take all their zeros in $\mathbb{R}$ ) in domains of $\mathbb{R}^{n}$ with prescribed boundary conditions under which we obtain a solution that has no blow ups in the interior of the considered domain. These encompass sone of the mentioned particular equations from physics as important subcases within a unified framework. Adapting the previous results from K. Gürlebeck, W. Sprößig [17], U. Kähler [18], F. Sommen, Xu Zhenyuan [29, 32, 33], J. Ryan [27] and others, we use adapted versions of the Teodorescu transform, the Cauchy transform, Plemelj projectors and the 
Bergman projection to represent the solutions of polynomial Dirac equations, in the particaular setting where the polynomial has all its zeros in $\mathbb{R}$. We extend the structural result obtained by W. Sprößig in [30] for polynomial Dirac equations of even degree associated to a particular choice of coefficients to the context of polynomial Dirac equations of any arbitrary degree with coefficients as mentioned before. In the case of dealing with polynomial Dirac equations of an odd degree one has to consider particular extra boundary conditions to obtain a solution. To do so we here involve the Plemelj projection of the function on the boundary. By imposing a boundary condition involving the Plemelj projection we determine in a natural way the choice of the singularity free part of the solutions - to be regular either in the inside or in the outside of the considered domain. Imposing the regular Plemelj projection $P$ as boundary condition we avoid blow-up effects of the solutions in the inside of the considered domain.

In the cases dealing with spherical and concentric annular domains in $\mathbb{R}^{n}$, we give fully explicit formulas for all integral kernels. These allow us to express the solutions to all these boundary value problems fully explicitly in terms of series over Bessel functions and homogeneous monogenic polynomials.

\section{Preliminaries}

Let $\left\{e_{1}, e_{2}, \ldots, e_{n}\right\}$ be the standard basis of $\mathbb{R}^{n}$ and $C l_{n}(\mathbb{R})$ be the associated real Clifford algebra in which $e_{i} e_{j}+e_{j} e_{i}=-2 \delta_{i j} e_{0}, \quad i, j=1, \cdots, n$, holds, $\delta_{i j}$ standing for the Kronecker symbol. Each $a \in C l_{n}(\mathbb{R})$ can be represented in the form $a=\sum_{A} a_{A} e_{A}$ with $a_{A} \in \mathbb{R}, A \subseteq\{1, \cdots, n\}, e_{A}=e_{l_{1}} e_{l_{2}} \cdots e_{l_{r}}$, where $1 \leq l_{1}<\cdots<l_{r} \leq n, \quad e_{\emptyset}=e_{0}=1$. The scalar part of $a, \operatorname{Sc}(a)$, is defined as the $a_{0}$ term. The Clifford conjugate of $a$ is defined by $\bar{a}=\sum_{A} a_{A} \bar{e}_{A}$, where $\bar{e}_{A}=\bar{e}_{l_{r}} \bar{e}_{l_{r-1}} \cdots \bar{e}_{l_{1}}$ and $\bar{e}_{j}=-e_{j}$ for $j=1, \cdots, n, \bar{e}_{0}=e_{0}=1$.

On $C l_{n}(\mathbb{R})$ one considers a standard (pseudo)norm defined by $\|a\|=\left(\sum_{A}\left|a_{A}\right|^{2}\right)^{1 / 2}$. Here $|\cdot|$ is the usual absolute value of the real number $a_{A}$.

Let $\Omega \subseteq \mathbb{R}^{n}$ be an open set and $\mathbf{x}:=x_{1} e_{1}+\cdots x_{n} e_{n}$ be a vector variable. Let $\mathbf{D}_{\mathbf{x}}=\sum_{j=1}^{n} \frac{\partial}{\partial x_{j}} e_{j}$ be the Euclidean Dirac operator. Let $\lambda \in \mathbb{R}$. A function $u: \Omega \rightarrow C l_{n}(\mathbb{R})$ that satisfies $\left(\mathbf{D}_{\mathbf{x}}-\lambda\right) u(\mathbf{x})=0$ is called $\lambda$-holomorphic or $\lambda$-monogenic. For details see for instance $[16,20,27,29,32]$ and elsewhere. In the particular case $\lambda=0$ one deals with the set of monogenic functions which are also often called hyperholomorphic or hypercomplex analytic. These are most closely related to complex holomorphic functions which are in the kernel of the Cauchy-Riemann operator, see for instance [5, 15].

\section{$3 \quad$ Hypercomplex integral operators}

In this section we introduce some well-known integral operators used for instance in $[16,17]$ and elsewhere. Following for instance [32], for general complex $\lambda \in \mathbb{C}$ 
the fundamental solution to $\left(\mathbf{D}_{\mathbf{x}}-\lambda\right) u(\mathbf{x})=0$ in $\mathbb{R}^{n}$ can be expressed as

$$
e_{\lambda}(\mathbf{x})= \begin{cases}\frac{\pi i}{A_{n} \Gamma(n / 2)}\left(\frac{\lambda}{2}\right)^{n / 2}\|\mathbf{x}\|^{1-n / 2}\left[H_{n / 2-1}^{(1)}(\lambda\|\mathbf{x}\|)-\frac{\mathbf{x}}{\|\mathbf{x}\|} H_{n / 2}^{(1)}(\lambda\|\mathbf{x}\|)\right], & \Im(\lambda)>0 \\ \frac{-\pi i}{A_{n} \Gamma(n / 2)}\left(\frac{\lambda}{2}\right)^{n / 2}\|\mathbf{x}\|^{1-n / 2}\left[H_{n / 2-1}^{(2)}(\lambda\|\mathbf{x}\|)-\frac{\mathbf{x}}{\|\mathbf{x}\|} H_{n / 2}^{(2)}(\lambda\|\mathbf{x}\|)\right], & \Im(\lambda)<0 \\ \frac{\pi}{A_{n} \Gamma(n / 2)}\left(\frac{\lambda}{2}\right)^{n / 2}\|\mathbf{x}\|^{1-n / 2}\left[Y_{n / 2-1}(\lambda\|\mathbf{x}\|)-\frac{\mathbf{x}}{\|\mathbf{x}\|} Y_{n / 2}(\lambda\|\mathbf{x}\|)\right], & \Im(\lambda)=0 .\end{cases}
$$

Here $A_{n}=2 \pi^{n / 2} / \Gamma(n / 2)$ denotes the 'surface area' of the unit ball in $\mathbb{R}^{n}$.

The functions $H^{(1)}$ and $H^{(2)}$ stand for the Hankel functions, defined by

$$
\begin{aligned}
& H_{\nu}^{(1)}(z)=J_{\nu}(z)+i Y_{\nu}(z), \quad \nu \in \frac{1}{2} \mathbb{N}, z \in \mathbb{C} \\
& H_{\nu}^{(2)}(z)=J_{\nu}(z)-i Y_{\nu}(z),
\end{aligned}
$$

see [13] for details. In the three-dimensional case $n=3$, the fundamental solution simplifies in the case where $\Im(\lambda)=0$ to the following expression, see [16]

$$
e_{\lambda}(\mathbf{x})=-\frac{\mathbf{x}}{4 \pi\|\mathbf{x}\|^{3}}[\cos (\lambda\|\mathbf{x}\|)-\|\mathbf{x}\| \lambda \sin (\lambda\|\mathbf{x}\|)]-\frac{\lambda \cos (\lambda\|\mathbf{x}\|)}{4 \pi\|\mathbf{x}\|} e_{0} .
$$

In the case $\lambda=0$ the expression $e_{\lambda}(\mathbf{x})$ simplifies to the usual Cauchy kernel function, i.e. $-\frac{\mathbf{x}}{\|\mathbf{x}\|^{n}}(n=3$ in the three-dimensional case). Suppose now that $\Omega \subset \mathbb{R}^{n}$ is a bounded domain with a piecewise smooth Liapunov boundary.

Furthermore, let us suppose in all that follows that $\lambda$ is purely real, i.e. $\lambda \in \mathbb{R}$. Then, following for example [17], the associated Teodorescu transform is given by

$$
\left[T_{\lambda} u\right](\mathbf{x})=-\int_{\Omega} e_{\lambda}(\mathbf{x}-\mathbf{y}) u(\mathbf{y}) d V(\mathbf{y}), \quad \mathbf{x} \in \mathbb{R}^{n}
$$

for $u \in C(\Omega)$.

The Cauchy transform is

$$
\left[F_{\lambda} u\right](\mathbf{x})=\int_{\partial \Omega} e_{\lambda}(\mathbf{x}-\mathbf{y}) \mathbf{n}(\mathbf{y}) u(\mathbf{y}) d S(\mathbf{y}), \quad \mathbf{x} \in \mathbb{R}^{n} \backslash \partial \Omega
$$

for $u \in C^{1}(\Omega) \cap C(\bar{\Omega})$.

Furthermore, one introduces the Plemelj projection operators by

$$
\left[P_{\lambda} u\right](\mathbf{x}):=\lim _{\mathbf{y} \rightarrow \mathbf{x}, \mathbf{y} \in \Omega}\left[F_{\lambda} u\right](\mathbf{y})=\frac{1}{2} u(\mathbf{x})+\frac{1}{A_{n}} P . V . \int_{\Gamma} e_{\lambda}(\mathbf{x}-\mathbf{w}) \mathbf{n}(\mathbf{w}) u(\mathbf{w}) d S(\mathbf{w})
$$

and

$$
\left[Q_{\lambda} u\right](\mathbf{x}):=-\lim _{\mathbf{y} \rightarrow \mathbf{x}, \mathbf{y} \notin \Omega}\left[F_{\lambda} u\right](\mathbf{y})=-\frac{1}{2} u(\mathbf{x})+\frac{1}{A_{n}} P . V . \int_{\Gamma} e_{\lambda}(\mathbf{x}-\mathbf{w}) \mathbf{n}(\mathbf{w}) u(\mathbf{w}) d S(\mathbf{w}),
$$


where $n$ is the usual outer normal field for the oriented surface integral, P.V. stands for the principal value in the sense of Cauchy and the limit is meant to be non-tangential, and $u$ is Hölder continuous on $\Gamma$ with a Hölder constant $\beta \in$ $(0,1]$. Notice that these entities can also be introduced completely analogously for the case where $\lambda$ is purely imaginary, see for instance [1].

More generally, we want to consider polynomial Dirac equations of the form

$$
P\left(\mathbf{D}_{\mathbf{x}}\right) u(\mathbf{x})=f(\mathbf{x})
$$

where $P\left(\mathbf{D}_{\mathbf{x}}\right)=a_{m} \mathbf{D}_{\mathbf{x}}^{m}+\cdots+a_{1} \mathbf{D}_{\mathbf{x}}+a_{0}$ is a polynomial expression of arbitrary degree $m \in \mathbb{N}$ and where $a_{0}, \ldots, a_{m}$ are arbitrary real coefficients such that $P\left(\mathbf{D}_{\mathbf{x}}\right)$ has real roots only. For convenience we write $P(\mathbf{D})$ for short when it is clear which variable is considered. For the fundamental theory of polynomial Dirac equations we refer the reader for instance to $[29,27]$.

An important feature of null-solutions to such a general polynomial Dirac equation is that the set of $L^{2}\left(\Omega, C l_{n}(\mathbb{R})\right)$ functions that are in $\operatorname{Ker} P(\mathbf{D})$ has a unique reproducing Bergman kernel, denoted by $B_{P(\mathbf{D})}(\mathbf{x}, \mathbf{y})$. This is shown in [10]. In the case where the polynomial has degree 1, J. Ryan has shown in [27] that this kernel exists for any domain with a piecewise smooth Liapunov boundary. In [10] we have shown that it does even exist for any kind of bounded or unbounded domain.

The Bergman kernel satisfies

$$
f(\mathbf{x})=\int_{\Omega} B_{P(\mathbf{D})}(\mathbf{x}, \mathbf{y}) f(\mathbf{y}) d V(\mathbf{y}) .
$$

The associated Bergman projector

$\mathbf{P}: L^{2}\left(\Omega, C l_{n}(\mathbb{R})\right) \rightarrow L^{2}\left(\Omega, C l_{n}(\mathbb{R})\right) \cap K \operatorname{Ker} P(\mathbf{D}), \quad[\mathbf{P} f](\mathbf{x}):=\int_{\Omega} B_{P(\mathbf{D})}(\mathbf{x}, \mathbf{y}) f(\mathbf{y}) d V(\mathbf{y})$

produces the ortho-projection from the $L^{2}\left(\Omega, C l_{n}(\mathbb{R})\right)$-space onto the so-called Bergman space $L^{2}\left(\Omega, C l_{n}(\mathbb{R})\right) \cap \operatorname{Ker} P(\mathbf{D})$, which is endowed with the inner product

$$
<f, g>:=\left(\int_{\Omega} \bar{f}(\mathbf{y}) g(\mathbf{y}) d V(\mathbf{y})\right) .
$$

In terms of the inner product, the reproducing property reads

$$
f(\mathbf{x})=<B(\cdot, \mathbf{x}), f>.
$$

The ortho-projection onto the complementary subspace of $L^{2}\left(\Omega, C l_{n}(\mathbb{R})\right)$ will be denoted by $\mathbf{Q}:=\mathbf{I}-\mathbf{P}$, where $\mathbf{I}$ is the identity operator.

In contrast to the Cauchy kernel appearing in the Cauchy and Teodorescu transform, the Bergman kernel depends on the domain. The determination of explicit formulas for the Bergman kernel is very difficult. However, its knowledge 
is very important if we want to solve Dirichlet type problems for polynomial Dirac equations analytically, as we shall see in the following section.

For the particular class of sub cases dealing with the first order system $\left(\mathbf{D}_{\mathbf{x}}-\right.$ $\lambda) f(\mathbf{x})=0$ with $\lambda \in \mathbb{C} \backslash\{0\})$ a formula for the Bergman kernel for the unit ball has been constructed in [6]. In the limit case $\lambda \rightarrow 0$ one obtains the formulas for the monogenic Bergman kernel in the unit ball, previously given in $[4,5,11]$.

In our recent papers $[9,10]$ we managed to establish explicit formulas for the Bergman kernel for polynomial Dirac equations for the case where $\Omega$ is the concentric annulus of radii $r=\mu \in[0,1[$ and $R=1$ and $\partial \Omega$ for its boundary. The limit case $\mu=0$ deals with the unit ball.

\section{Boundary value problems of polynomial Dirac equations}

In this section we give an application of the explicit formulas for the Bergman kernels that were developed in our previous papers $[9,10]$. We show how these are useful and needed to set up an explicit representation formulas for general Dirichlet type boundary value problems $P(\mathbf{D}) u=f$ inside the annulus and with prescribed data on the boundary parts of the annulus. Here, $P$ may again be a real polynomial expression in $\mathbf{D}$ with the additional restriction that all its roots are real. Without loss of generality we suppose that the leading coefficient is 1 , that means $P(\mathbf{D})$ has the form $\mathbf{D}^{m}+a_{m-1} \mathbf{D}^{m-1}+\cdots a_{1} \mathbf{D}+a_{0}$. Remember that any arbitrary real polynomial of degree $m$ can be decomposed into $m$ linear factors $\left(\mathbf{D}-\lambda_{1}\right) \cdots\left(\mathbf{D}-\lambda_{m}\right)$. The values $\lambda_{1}, \lambda_{2}, \cdots, \lambda_{m}$ are either real numbers or pairs of complex conjugated numbers and do not need to be all pairwise distinct. Some of them might be zero. The case of existemce of pairs of complex conjugated numbers will not be considered in this paper.

Let us further consider the case where $\Omega \subset \mathbb{R}^{n}$ is an arbitrary bounded domain that has a piecewise smooth Liapunov denoted by $\partial \Omega$ or $\Gamma$ for short.

The function $f$ on the right-hand side is supposed to be an element of the Lebesgue space $L^{2}\left(\Omega, C l_{n}(\mathbb{R})\right)$. An important question is to know which boundary conditions can be imposed on the equation $P(\mathbf{D}) u=f$ such that this system has a solution, in particular, a unique solution. A further task is to give an explicit representation formula for these solutions. We shall see that we can express the solutions in terms of the integral operators described above. In the cases of annular shaped domains and of the ball we can set up explicit representation formulas using the formulas for the Bergman kernels that were computed in the earlier papers $[9,10]$.

Before we start, we briefly reconsider mapping properties of several operators, which will be used in the following. Let $\lambda$ and $\mu$ be both from $\mathbb{R}$. So let us first suppose that $\Omega \subset \mathbb{R}^{n}$ is an arbitrary bounded domain with a piecewise smooth 
Liapunov boundary. Then, following for instance $[2,17]$, we have

$$
\begin{aligned}
T_{\lambda} & : \quad W_{p}^{k}\left(\Omega, C l_{n}(\mathbb{C})\right) \longrightarrow W_{p}^{k+1}\left(\Omega, C l_{n}(\mathbb{C})\right) \\
F_{\lambda} & : \quad W_{p}^{k-\frac{1}{p}}\left(\Gamma, C l_{n}(\mathbb{C})\right) \longrightarrow W_{p}^{k}\left(\Omega, C l_{n}(\mathbb{C})\right) \cap \operatorname{Ker}(\mathbf{D}-\lambda) \\
\mathbf{P}_{\lambda} & : \quad L^{2}\left(\Omega, C l_{n}(\mathbb{C})\right) \longrightarrow \operatorname{Ker}(\mathbf{D}-\lambda) \cap L^{2}\left(\Omega, C l_{n}(\mathbb{C})\right) \\
\mathbf{Q}_{\lambda} & : \quad L^{2}\left(\Omega, C l_{n}(\mathbb{C})\right) \longrightarrow\left(\mathbf{D}-\lambda^{\sharp}\right) W_{2}^{1}\left(\Omega, C l_{n}(\mathbb{C})\right) \cap L^{2}\left(\Omega, C l_{n}(\mathbb{C})(4)\right. \\
\left(\operatorname{tr}_{\Gamma} T_{\mu} F_{\lambda}\right) & : \quad i m P_{\lambda} \cap W_{2}^{k+\frac{1}{2}}\left(\Gamma, C l_{n}(\mathbb{C})\right) \longrightarrow i m Q_{\mu} \cap W_{2}^{k+\frac{3}{2}}\left(\Gamma, C l_{n}(\mathbb{C})\right) .
\end{aligned}
$$

Here, $\operatorname{tr}_{\Gamma}$ denotes the trace operator, which is just the restriction of its argument to the boundary $\Gamma$.

We start with an important theorem, which we will use again and again in the cause of the paper:

Corollary 1 (Borel-Pompeiu formula). Let $\Omega \subseteq \mathbb{R}^{n}$ be a domain which is bounded by a piecewise smooth Liapunov surface $\Gamma$. Then for each $u \in C^{1}\left(\Omega, C l_{n}(\mathbb{R})\right) \cap$ $C\left(\bar{\Omega}, C l_{n}(\mathbb{R})\right)$ and $\lambda \in \mathbb{R}$ holds

$$
\left(F_{\lambda} u\right)(z)+\left(T_{\lambda} \mathbf{D}_{\lambda} u\right)(z)= \begin{cases}\left(\mathbf{D}_{\lambda} T_{\lambda} u\right)(z)=u(z), & z \in \Omega \\ 0, & z \in \mathbb{R}^{n} \backslash \Omega\end{cases}
$$

A proof can be found in [17].

First we look at the simplest system of a polynomial Dirac equation of degree 1.

Theorem 1. Let $\lambda \in \mathbb{R}$. Let $\Omega \subset \mathbb{R}^{n}$ be an arbitrary bounded domain with a piecewise smooth Liapunov boundary. Let $f \in W_{2}^{k+1}\left(\Omega, C l_{n}(\mathbb{R})\right)$ and $g \in$ $W_{2}^{k+3 / 2}\left(\Gamma, C l_{n}(\mathbb{R})\right)$ and $g \in i m P_{\lambda}$. Then the solutions of the system

$$
\begin{aligned}
\left(\mathbf{D}_{\mathbf{x}}-\lambda\right) u(\mathbf{x}) & =f(\mathbf{x}) \text { in } \Omega \\
P_{\lambda} u(\mathbf{x}) & =g(\mathbf{x}) \text { on } \partial \Omega
\end{aligned}
$$

have the form $h+T_{\lambda} f \in W_{2}^{k+2}\left(\Omega, C l_{n}(\mathbb{R})\right)$, where $h$ is a $W_{2}^{k+2}\left(\Omega, C l_{n}(\mathbb{R})\right)$ extension of $g$.

Proof. Suppose that $h$ is a $W_{2}^{k+2}\left(\Omega, C l_{n}(\mathbb{R})\right)$ extension of $g$ on $\bar{\Omega}$. Then BorelPompeiu's formula yields:

$$
\left[F_{\lambda} u\right](\mathbf{x})+\left[T_{\lambda}\left(\mathbf{D}_{\mathbf{x}}-\lambda\right)\right] u(\mathbf{x})=u(\mathbf{x}) \quad \text { in } \Omega .
$$

Hence, $h(\mathbf{x})=\left[F_{\lambda} u\right](\mathbf{x})=u(\mathbf{x})-\left[T_{\lambda}\left(\mathbf{D}_{\mathbf{x}}-\lambda\right)\right] u(\mathbf{x})=u(\mathbf{x})-\left[T_{\lambda} f\right](\mathbf{x})$. Therefore $u=h+\left[T_{\lambda} f\right]$.

In regard of the mapping properties of $T_{\lambda}$ (see (1)), we have $\left[T_{\lambda} f\right] \in W_{2}^{k+2}\left(\Omega, C l_{n}(\mathbb{R})\right.$ ) and thus $u \in W_{2}^{k+2}\left(\Omega, C l_{n}(\mathbb{R})\right)$. 
It is very well-known, see for instance [16, 17] and in many other textbooks on classical harmonic analysis that the analogous second order boundary value problem

$$
\begin{aligned}
(\mathbf{D}-\lambda)(\mathbf{D}-i \lambda) u(\mathbf{x}) & =f(\mathbf{x}) \text { in } \Omega \\
u(\mathbf{x}) & =g(\mathbf{x}) \text { on } \partial \Omega
\end{aligned}
$$

where $i$ stands for the classical complex imaginary unit, has always a solution for $g \in i m P_{\lambda}$. Following e.g. [16] a solution to this system can be represented in the form

$$
u=F_{i \lambda} g+T_{i \lambda} \mathbf{P}_{\lambda}(\mathbf{D}-i \lambda) h+T_{i \lambda}\left(\mathbf{I}-\mathbf{P}_{\lambda}\right) T_{\lambda} f .
$$

Here $\mathbf{P}_{\lambda}$ is the Bergman projection associated to the first order operator $P(\mathbf{D})=$ $\mathbf{D}-\lambda$. In the case where $\lambda$ is real, this is the classical Helmholtz equation. Now we consider two theorems presented in [30] and present a complete detailed proof of them, since this has not been done in [30, 17], because we need the technique and steps of these proofs in order to prove the main result (Theorem 4) of this section and to make the paper self-contained.

For the following theorem, we introduce the following operators:

$$
\begin{aligned}
\mathbf{P}_{\lambda \mu} & :=F_{\lambda}\left(\operatorname{tr}_{\Gamma} T_{\mu} F_{\lambda}\right)^{-1} \operatorname{tr}_{\Gamma} T_{\mu} \\
\mathbf{Q}_{\lambda \mu} & :=\mathbf{I}-\mathbf{P}_{\lambda \mu}
\end{aligned}
$$

for any $\lambda, \mu \in \mathbb{R}$.

Note that $\left(\operatorname{tr}_{\Gamma} T_{\mu} F_{\lambda}\right)^{-1} \operatorname{tr}_{\Gamma}$ and thus $\mathbf{P}_{\lambda \mu}$ only exist if the boundary value problem

$$
\begin{aligned}
\mathbf{D}_{\lambda} \mathbf{D}_{\mu} u & =0 & & \text { in } \Omega \\
u & =g & & \text { on } \Gamma,
\end{aligned}
$$

is solvable.

From now on, we assume this problem to be uniquely solvable.

From [17] (see page 147) we have the following

Lemma 1. For $m \geq 1$ the projection $\mathbf{P}_{\lambda \mu}$ is a continuous operator within the Sobolev space $W_{2}^{m}\left(\Omega, C l_{n}(\mathbb{R})\right)$.

Thus we also have

$$
\mathbf{Q}_{\lambda \mu}: W_{2}^{m}\left(\Omega, C l_{n}(\mathbb{R})\right) \longrightarrow W_{2}^{m}\left(\Omega, C l_{n}(\mathbb{R})\right) .
$$

Theorem 2. Let $f \in W_{2}^{m}\left(\Omega, C l_{n}(\mathbb{R})\right), g \in W_{2}^{m+\frac{3}{2}}\left(\Gamma, C l_{n}(\mathbb{R})\right) \cap i m P_{\lambda}$ and the problem in (6) be solvable. In the case that $\lambda^{2}$ and $\mu^{2}$ are no eigenvalues of the negative Laplacian $-\Delta$, the boundary value problem

$$
\begin{aligned}
\left(\mathbf{D}_{\mathbf{x}}-\lambda\right)\left(\mathbf{D}_{\mathbf{x}}-\mu\right) u(\mathbf{x}) & =f(\mathbf{x}) & & \text { in } \Omega \\
u(\mathbf{x}) & =g(\mathbf{x}) & & \text { on } \Gamma,
\end{aligned}
$$


has a solution $u \in W_{2}^{m+2}\left(\Omega, C l_{n}(\mathbb{R})\right)$ and can be represented by the formula

$$
u=F_{\mu} g+T_{\mu} F_{\lambda}\left(\operatorname{tr}_{\Gamma} T_{\mu} F_{\lambda}\right)^{-1} Q_{\mu} g+T_{\mu} \mathbf{Q}_{\lambda \mu} T_{\lambda} f .
$$

Proof. Firstly, we have

$$
\begin{aligned}
(\mathbf{D}-\mu) u & =\underbrace{(\mathbf{D}-\mu) F_{\mu}}_{=0} g+\underbrace{(\mathbf{D}-\mu) T_{\mu}}_{=\mathbf{I}} F_{\lambda}\left(\operatorname{tr}_{\Gamma} T_{\mu} F_{\lambda}\right)^{-1} Q_{\mu} g+\underbrace{(\mathbf{D}-\mu) T_{\mu}}_{=\mathbf{I}} \mathbf{Q}_{\lambda \mu} T_{\lambda} f \\
& =F_{\lambda}\left(t_{\Gamma} T_{\mu} F_{\lambda}\right)^{-1} Q_{\mu} g+\mathbf{Q}_{\lambda \mu} T_{\lambda} f,
\end{aligned}
$$

and

$$
\begin{aligned}
(\mathbf{D}-\lambda)(\mathbf{D}-\mu) u & =(\mathbf{D}-\lambda) F_{\lambda}\left(\operatorname{tr}_{\Gamma} T_{\mu} F_{\lambda}\right)^{-1} Q_{\mu} g+D_{\lambda} \mathbf{Q}_{\lambda \mu} T_{\lambda} f \\
& =(\mathbf{D}-\lambda)\left(\mathbf{I}-\mathbf{P}_{\lambda \mu}\right) T_{\lambda} f \\
& =(\mathbf{D}-\lambda) T_{\lambda} f-\underbrace{\mathbf{P}_{\lambda \mu}(\mathbf{D}-\lambda)}_{=0} T_{\lambda} f \\
& =f,
\end{aligned}
$$

since $i m \mathbf{P}_{\lambda \mu} \subseteq\left\{f: \Omega \rightarrow C l_{n}(\mathbb{R}) ;(\mathbf{D}-\lambda)(\mathbf{D}-\mu) f=0\right\}$.

For the boundary condition one computes

$$
\operatorname{tr}_{\Gamma} T_{\mu} \mathbf{Q}_{\lambda \mu} T_{\lambda} f=0
$$

and

$$
\operatorname{tr}_{\Gamma} F_{\mu} g+\operatorname{tr}_{\Gamma} T_{\mu} F_{\lambda}\left(\operatorname{tr}_{\Gamma} T_{\mu} F_{\lambda}\right)^{-1} Q_{\mu} g=P_{\mu} g+Q_{\mu} g=g .
$$

The first equation is hereby shown as follows:

From the definition of $\mathbf{P}_{\lambda \mu}$ and $\mathbf{Q}_{\lambda \mu}$ we have

$$
\begin{aligned}
\operatorname{tr}_{\Gamma} T_{\mu} \mathbf{Q}_{\lambda \mu} T_{\lambda} f & =\operatorname{tr}_{\Gamma} T_{\mu} T_{\lambda} f-\operatorname{tr}_{\Gamma} T_{\mu} \mathbf{P}_{\lambda \mu} T_{\lambda} f \\
& =\operatorname{tr}_{\Gamma} T_{\mu} T_{\lambda} f-\underbrace{\operatorname{tr}_{\Gamma} T_{\mu} F_{\lambda}\left(t_{\Gamma} T_{\mu} F_{\lambda}\right)^{-1}}_{=\mathbf{I}} \operatorname{tr}_{\Gamma} T_{\mu} T_{\lambda} f \\
& =0 .
\end{aligned}
$$

Since $g \in W^{m+\frac{3}{2}}\left(\Gamma, C l_{n}(\mathbb{R})\right)$ it follows by applying property (2) that $F_{\mu} g \in$ $W_{2}^{m+2}\left(\Omega, C l_{n}(\mathbb{R})\right)$, after inserting $k=m+2$ and $p=2$ into $(2)$.

Property (5) in turn leads to $\left(\operatorname{tr}_{\Gamma} T_{\mu} F_{\lambda}\right)^{-1} Q_{\mu} g \in W_{2}^{m+\frac{1}{2}}\left(\Omega, C l_{n}(\mathbb{R})\right)$ and (1) and (2) to

$$
T_{\mu} F_{\lambda}\left(\operatorname{tr}_{\Gamma} T_{\mu} F_{\lambda}\right)^{-1} Q_{\mu} g \in W^{m+\frac{1}{2}+\frac{1}{2}+1}\left(\Omega, C l_{n}(\mathbb{R})\right)=W_{2}^{m+2}\left(\Omega, C l_{n}(\mathbb{R})\right) .
$$

At last with (1) and (8), we have

$$
T_{\mu} \mathbf{Q}_{\lambda \mu} T_{\lambda} f \in W_{2}^{m+1+1}\left(\Omega, C l_{n}(\mathbb{R})\right)=W_{2}^{m+2}\left(\Omega, C l_{n}(\mathbb{R})\right) .
$$

So $u \in W_{2}^{m+2}\left(\Omega, C l_{n}(\mathbb{R})\right)$. This completes the proof. 
More generally, W. Sprößig was able to prove a more general result for boundary value problems of even order $n=2 m$. We can directly adapt the result from [30] (see also [17] p. 161) to the case where the pairs $\left(\lambda_{i}, \mu_{i}\right) i=1, \ldots, m$ are real numbers as follows:

Theorem 3. Let $\Omega \subset \mathbb{R}^{n}$ be a bounded domain with a piecewise smooth Liapunov boundary $\Gamma$. Let $f \in L_{2}\left(\Omega, C l_{n}(\mathbb{R})\right), g_{i} \in W_{2}^{2 m-\frac{4 i+1}{2}}\left(\Gamma, C l_{n}(\mathbb{R})\right)$ for $i=0, \cdots, m-1$ and $\lambda_{j}, \mu_{j},(j=1, \cdots, m)$ be $2 m$ real numbers in $\mathbb{R}$ whose squares are not eigenvalues of the negative Laplacian $-\Delta$. Then the solution of the Dirichlet type problem

$$
\prod_{i=1}^{m}\left(\mathbf{D}_{\mathbf{x}}-\lambda_{i}\right)\left(\mathbf{D}_{\mathbf{x}}-\mu_{i}\right) u(\mathbf{x})=f(\mathbf{x}) \text { in } \Omega
$$

with the boundary conditions

$u(\mathbf{x})=g_{0}(\mathbf{x}),\left(\mathbf{D}_{\mathbf{x}}-\lambda_{1}\right)\left(\mathbf{D}_{\mathbf{x}}-\mu_{1}\right) u(\mathbf{x})=g_{1}(\mathbf{x}), \ldots, \prod_{i=1}^{m-1}\left(\mathbf{D}_{\mathbf{x}}-\lambda_{i}\right)\left(\mathbf{D}_{\mathbf{x}}-\mu_{i}\right) u(\mathbf{x})=g_{m-1}(\mathbf{x})$

on $\Gamma$ has the form

$$
\begin{aligned}
u & =F_{\mu_{1}} g_{0}+T_{\mu_{1}} F_{\lambda_{1}}\left(\operatorname{tr}_{\Gamma} T_{\mu_{1}} F_{\lambda_{1}}\right)^{-1} Q_{\mu_{1}} g_{0} \\
& \left.+T_{\mu_{1}} \mathbf{Q}_{\lambda_{1} \mu_{1}} T_{\lambda_{1}}\left(F_{\mu_{2}} g_{1}+T_{\mu_{2}} F_{\lambda_{2}}\left(\operatorname{tr}_{\Gamma} T_{\mu_{2}} F_{\lambda_{2}}\right)\right)^{-1} Q_{\mu_{2}} g_{1}\right)+ \\
& \vdots \\
& +\prod_{j=1}^{m-1} T_{\mu_{j}} \mathbf{Q}_{\lambda_{j} \mu_{j}} T_{\lambda_{j}}\left(F_{\mu_{m}} g_{m-1}+T_{\mu_{m}} F_{\lambda_{m}}\left(\operatorname{tr}_{\Gamma} T_{\mu_{m}} F_{\lambda_{m}}\right)^{-1} Q_{\mu_{m}} g_{m-1}\right)+ \\
& +\prod_{j=1}^{m} T_{\mu_{j}} \mathbf{Q}_{\lambda_{j} \mu_{j}} T_{\lambda_{j}} f .
\end{aligned}
$$

Here we put $\mathbf{Q}_{\lambda_{j} \mu_{j}}:=\mathbf{I}-\mathbf{P}_{\lambda_{j} \mu_{j}}$ where $\mathbf{P}_{\lambda_{j} \mu_{j}}$ is the Bergman projection from $L_{2}\left(\Omega, C l_{n}(\mathbb{R})\right)$ onto $L_{2}\left(\Omega, C l_{n}(\mathbb{R})\right) \cap \operatorname{Ker}\left(\mathbf{D}_{\mathbf{x}}-\lambda_{j}\right)\left(\mathbf{D}_{\mathbf{x}}-\mu_{j}\right)$.

Proof. We proof the representation of $u$ with induction over $m$. The case $m=1$ is just treated in Theorem 2. So consider now $m \geq 2$. Putting

$$
\tilde{u}(\mathbf{x}):=\prod_{i=1}^{m-1}\left(\mathbf{D}_{\mathbf{x}}-\lambda_{i}\right)\left(\mathbf{D}_{\mathbf{x}}-\mu_{i}\right) u(\mathbf{x}),
$$

then we obtain the system

$$
\begin{aligned}
\left(\mathbf{D}_{\mathbf{x}}-\lambda_{m}\right)\left(\mathbf{D}_{\mathbf{x}}-\mu_{m}\right) \tilde{u}(\mathbf{x}) & =f(\mathbf{x}) \text { in } \Omega \\
\tilde{u}(\mathbf{x}) & =g_{m-1}(\mathbf{x}) \text { at } \Gamma .
\end{aligned}
$$

Theorem 2 yields

$\tilde{u}=F_{\mu_{m}} g_{m-1}+T_{\mu_{m}} F_{\lambda_{m}}\left(\operatorname{tr}_{\Gamma} T_{\mu_{m}} F_{\lambda_{m}}\right)^{-1} Q_{\mu_{m}} g_{m-1}+T_{\mu_{m}} \mathbf{Q}_{\lambda_{m} \mu_{m}} T_{\lambda_{m}} f \in W_{2}^{2}\left(\Omega, C l_{n}(\mathbb{R})\right)$. 
According to the definition, $\tilde{u}$ can be substituted by $\prod_{i=1}^{m-1}\left(\mathbf{D}_{\mathbf{x}}-\lambda_{i}\right)\left(\mathbf{D}_{\mathbf{x}}-\mu_{i}\right) u$.

So we can set up a new system

$\prod_{i=1}^{m-1}\left(\mathbf{D}_{\mathbf{x}}-\lambda_{i}\right)\left(\mathbf{D}_{\mathbf{x}}-\mu_{i}\right) u=\tilde{f}:=F_{\mu_{m}} g_{m-1}+T_{\mu_{m}} F_{\lambda_{m}}\left(t_{\Gamma} T_{\mu_{m}} F_{\lambda_{m}}\right)^{-1} Q_{\mu_{m}} g_{m-1}+T_{\mu_{m}} \mathbf{Q}_{\lambda_{m} \mu_{m}} T_{\lambda_{m}} f$

with the boundary conditions

$$
u=g_{0},\left(\mathbf{D}_{\mathbf{x}}-\lambda_{1}\right)\left(\mathbf{D}_{\mathbf{x}}-\mu_{1}\right) u=g_{1}, \ldots, \prod_{i=1}^{m-2}\left(\mathbf{D}_{\mathbf{x}}-\lambda_{i}\right)\left(\mathbf{D}_{\mathbf{x}}-\mu_{i}\right) u=g_{m-2}
$$

on $\Gamma$. Now we can apply the induction hypothesis and get

$$
\begin{aligned}
u & =F_{\mu_{1}} g_{0}+T_{\mu_{1}} F_{\lambda_{1}}\left(\operatorname{tr}_{\Gamma} T_{\mu_{1}} F_{\lambda_{1}}\right)^{-1} Q_{\mu_{1}} g_{0} \\
& \left.+T_{\mu_{1}} \mathbf{Q}_{\lambda_{1} \mu_{1}} T_{\lambda_{1}}\left(F_{\mu_{2}} g_{1}+T_{\mu_{2}} F_{\lambda_{2}}\left(\operatorname{tr}_{\Gamma} T_{\mu_{2}} F_{\lambda_{2}}\right)\right)^{-1} Q_{\mu_{2}} g_{1}\right)+ \\
& \vdots \\
& +\prod_{j=1}^{m-2} T_{\mu_{j}} \mathbf{Q}_{\lambda_{j} \mu_{j}} T_{\lambda_{j}}\left(F_{\mu_{m-1}} g_{m-2}+T_{\mu_{m-1}} F_{\lambda_{m-1}}\left(\operatorname{tr}_{\Gamma} T_{\mu_{m-1}} F_{\lambda_{m-1}}\right)^{-1} Q_{\mu_{m-1}} g_{m-2}\right)+ \\
& +\prod_{j=1}^{m-1} T_{\mu_{j}} \mathbf{Q}_{\lambda_{j} \mu_{j}} T_{\lambda_{j}} \tilde{f} .
\end{aligned}
$$

Writing out $\tilde{f}$ as $\tilde{f}=F_{\mu_{m}} g_{m-1}+T_{\mu_{m}} F_{\lambda_{m}}\left(\operatorname{tr}_{\Gamma} T_{\mu_{m}} F_{\lambda_{m}}\right)^{-1} Q_{\mu_{m}} g_{m-1}+T_{\mu_{m}} \mathbf{Q}_{\lambda_{m} \mu_{m}} T_{\lambda_{m}} f$ leads to

$$
\prod_{j=1}^{m-1} T_{\mu_{j}} \mathbf{Q}_{\lambda_{j} \mu_{j}} T_{\lambda_{j}}\left(\left(F_{\mu_{m}} g_{m-1}+T_{\mu_{m}} F_{\lambda_{m}}\left(\operatorname{tr}_{\Gamma} T_{\mu_{m}} F_{\lambda_{m}}\right)^{-1} Q_{\mu_{m}} g_{m-1}\right)+T_{\mu_{m}} \mathbf{Q}_{\lambda_{m} \mu_{m}} T_{\lambda_{m}} f\right)
$$

which equals

$$
\begin{aligned}
& \prod_{j=1}^{m-1} T_{\mu_{j}} \mathbf{Q}_{\lambda_{j} \mu_{j}} T_{\lambda_{j}}\left(\left(F_{\mu_{m}} g_{m-1}+T_{\mu_{m}} F_{\lambda_{m}}\left(\operatorname{tr}_{\Gamma} T_{\mu_{m}} F_{\lambda_{m}}\right)^{-1} Q_{\mu_{m}} g_{m-1}\right)\right) \\
+ & \prod_{j=1}^{m} T_{\mu_{j}} \mathbf{Q}_{\lambda_{j} \mu_{j}} T_{\lambda_{j}} f .
\end{aligned}
$$

These are the last missing terms for the proposed representation of $u$.

Concerning the smoothness: By induction, all summands up to the last two are already elements of $W_{2}^{2 m-2}\left(\Omega, C l_{n}(\mathbb{R})\right)$. However, since we assume that $g_{i} \in W^{2 m-\frac{4 i+1}{2}}\left(\Gamma, C l_{n}(\mathbb{R})\right)$ and not $g_{i} \in W^{2(m-1)-\frac{4 i+1}{2}}\left(\Gamma, C l_{n}(\mathbb{R})\right)$, the summands are also elements of $W_{2}^{2 m}\left(\Omega, C l_{n}(\mathbb{R})\right)$.

As described in the proof to Theorem 2, the application of the operator $T_{\mu_{j}} \mathbf{Q}_{\lambda_{j} \mu_{j}} T_{\lambda_{j}}$ implies the weak differentiability of its argument. Thus is

$$
\prod_{j=1}^{m} T_{\mu_{j}} \mathbf{Q}_{\lambda_{j} \mu_{j}} T_{\lambda_{j}} f \in W^{2 m}\left(\Omega, C l_{n}(\mathbb{R})\right) .
$$


The application of Theorem 2 lead to

$$
\left(F_{\mu_{m}} g_{m-1}+T_{\mu_{m}} F_{\lambda_{m}}\left(\operatorname{tr}_{\Gamma} T_{\mu_{m}} F_{\lambda_{m}}\right)^{-1} Q_{\mu_{m}} g_{m-1}\right) \in W_{2}^{2}\left(\Omega, C l_{n}(\mathbb{R})\right),
$$

and thus we have

$$
\prod_{j=1}^{m} T_{\mu_{j}} \mathbf{Q}_{\lambda_{j} \mu_{j}} T_{\lambda_{j}}\left(F_{\mu_{m}} g_{m-1}+T_{\mu_{m}} F_{\lambda_{m}}\left(\operatorname{tr}_{\Gamma} T_{\mu_{m}} F_{\lambda_{m}}\right)^{-1} Q_{\mu_{m}} g_{m-1}\right) \in W_{2}^{2 m}\left(\Omega, C l_{n}(\mathbb{R})\right) .
$$

So, all summands are elements of the space $W_{2}^{2 m}\left(\Omega, C l_{n}(\mathbb{R})\right)$ and so is $u$.

Our aim is now to prove a similar result for the case where the number of values $\lambda_{i}$ is odd. Notice that we cannot expect that a system of the form

$$
\left(\mathbf{D}_{\mathbf{x}}-\eta\right)\left[\prod_{i=1}^{m}\left(\mathbf{D}_{\mathbf{x}}-\lambda_{i}\right)\left(\mathbf{D}_{\mathbf{x}}-\mu_{i}\right)\right] u(\mathbf{x})=f(\mathbf{x}) \text { in } \Omega
$$

with the boundary conditions

$u(\mathbf{x})=g_{0}(\mathbf{x}),\left(\mathbf{D}_{\mathbf{x}}-\lambda_{1}\right)\left(\mathbf{D}_{\mathbf{x}}-\mu_{1}\right) u(\mathbf{x})=g_{1}(\mathbf{x}), \ldots, \prod_{i=1}^{m-1}\left(\mathbf{D}_{\mathbf{x}}-\lambda_{i}\right)\left(\mathbf{D}_{\mathbf{x}}-\mu_{i}\right) u(\mathbf{x})=g_{m-1}(\mathbf{x})$

and

$$
\prod_{i=1}^{m}\left(\mathbf{D}_{\mathbf{x}}-\lambda_{i}\right)\left(\mathbf{D}_{\mathbf{x}}-\mu_{i}\right) u(\mathbf{x})=g_{m}(\mathbf{x})
$$

on $\Gamma$ will have a solution. In the case $m=0$ which corresponds to the first order boundary value problem

$$
\begin{aligned}
(\mathbf{D}-\eta) u(\mathbf{x}) & =f(\mathbf{x}) \text { in } \Omega \\
u(\mathbf{x}) & =g_{0}(\mathbf{x}) \text { at } \partial \Omega
\end{aligned}
$$

the concrete example given above after Theorem 1 provides us with a concrete counter example.

One way to ensure existence is to replace the last condition (9) by considering the Plemelj projection of that expression.

Now we can prove the main result of this section:

Theorem 4. Let $\Omega \subset \mathbb{R}^{n}$ be a bounded domain with a piecewise smooth Liapunov boundary $\Gamma$. Let $m$ be a non-negative integer. Let $f \in L^{2}\left(\Omega, C l_{n}(\mathbb{R})\right)$ and that $g_{i} \in W_{2}^{2 m+2-\frac{4 i+1}{2}}\left(\Gamma, C l_{n}(\mathbb{R})\right), g \in i m P_{\eta}$. Suppose that $\eta$ and $\lambda_{i}, \mu_{i}$ for $i \leq m$ are arbitrary real numbers in $\mathbb{R}$ and that none of the squares of them is an eigenvalue of $-\Delta$. Then the $(2 m+1)$ order system

$$
\left(\mathbf{D}_{\mathbf{x}}-\eta\right)\left[\prod_{i=1}^{m}\left(\mathbf{D}_{\mathbf{x}}-\lambda_{i}\right)\left(\mathbf{D}_{\mathbf{x}}-\mu_{i}\right)\right] u(\mathbf{x})=f(\mathbf{x}) \text { in } \Omega
$$


with the boundary conditions

$u(\mathbf{x})=g_{0}(\mathbf{x}),\left(\mathbf{D}_{\mathbf{x}}-\lambda_{1}\right)\left(\mathbf{D}_{\mathbf{x}}-\mu_{1}\right) u(\mathbf{x})=g_{1}(\mathbf{x}), \ldots, \prod_{i=1}^{m-1}\left(\mathbf{D}_{\mathbf{x}}-\lambda_{i}\right)\left(\mathbf{D}_{\mathbf{x}}-\mu_{i}\right) u(\mathbf{x})=g_{m-1}(\mathbf{x})$

and

$$
P_{\eta}\left[\prod_{i=1}^{m}\left(\mathbf{D}_{\mathbf{x}}-\lambda_{i}\right)\left(\mathbf{D}_{\mathbf{x}}-\mu_{i}\right)\right] u(\mathbf{x})=g_{m}(\mathbf{x})
$$

on $\Gamma$ is solvable and its solution can be represented in the form

$$
\begin{aligned}
u= & F_{\mu_{1}} g_{0}+T_{\mu_{1}} F_{\lambda_{1}}\left(\operatorname{tr}_{\Gamma} T_{\mu_{1}} F_{\lambda_{1}}\right)^{-1} Q_{\mu_{1}} g_{0} \\
& +T_{\mu_{1}} \mathbf{Q}_{\lambda_{1} \mu_{1}} T_{\lambda_{1}}\left(T_{\mu_{2}} F_{\lambda_{2}}\left(\operatorname{tr}_{\Gamma} T_{\mu_{2}} F_{\lambda_{2}}\right)^{-1} Q_{\mu_{2}} g_{1}\right)+ \\
& \cdots \\
& \Pi_{j=1}^{m-1} T_{\mu_{j}} \mathbf{Q}_{\lambda_{j} \mu_{j}} T_{\lambda_{j}}\left(T_{\mu_{m}} F_{\lambda_{m}}\left(\operatorname{tr}_{\Gamma} T_{\mu_{m}} F_{\lambda_{m}}\right)^{-1} Q_{\mu_{m}} g_{m-1}\right)+ \\
& \Pi_{j=1}^{m} T_{\mu_{j}} \mathbf{Q}_{\lambda_{j} \mu_{j}} T_{\lambda_{j}}\left(h+T_{\eta} f\right) .
\end{aligned}
$$

where $h$ is a $W_{2}^{\frac{3}{2}}\left(\Omega, C l_{n}(\mathbb{R})\right)$ extension of $g_{m}$ from $\Gamma$ to $\Omega$.

Proof. We prove this theorem by mathematical induction over $m$. The case $m=0$ corresponds to the result from Theorem 1 and represents the inhomogeneous time-harmonic Maxwell system with no blow ups in the interior of the domain. Now let $m \geq 0$ be some arbitrary integer. We define the function $\tilde{u}(\mathbf{x})=\left[\prod_{i=1}^{m}\left(\mathbf{D}_{\mathbf{x}}-\lambda_{i}\right)\left(\mathbf{D}_{\mathbf{x}}-\mu_{i}\right)\right] u(\mathbf{x})$ that respects the corresponding boundary conditions. From Theorem 2 we know that the solutions to the system

$$
\begin{array}{rll}
(\mathbf{D}-\eta) \tilde{u}(\mathbf{x}) & =f(\mathbf{x}) & \text { in } \Omega \\
P_{\eta} \tilde{u}(\mathbf{x}) & =g(\mathbf{x}) & \text { on } \Gamma,
\end{array}
$$

where we now put $\tilde{u}(\mathbf{x})=\left[\prod_{i=1}^{m}\left(\mathbf{D}_{\mathbf{x}}-\lambda_{i}\right)\left(\mathbf{D}_{\mathbf{x}}-\mu_{i}\right)\right] u(\mathbf{x})$, are of the form $h(\mathbf{x})+$ $\left[T_{\eta} f\right](\mathbf{x})$ where $h$ is a $W_{2}^{\frac{3}{2}}\left(\Omega, C l_{n}(\mathbb{R})\right)$-extension of $g$ from $\Gamma$ to $\Omega$. This leads to the $2 m$ order system of the form

$$
\tilde{u}(\mathbf{x})=\left[\prod_{i=1}^{m}\left(\mathbf{D}_{\mathbf{x}}-\lambda_{i}\right)\left(\mathbf{D}_{\mathbf{x}}-\mu_{i}\right)\right] u(\mathbf{x})=h(\mathbf{x})+\left[T_{\eta} f\right](\mathbf{x})
$$

with the corresponding boundary value conditions. From Theorem 3 we may now directly infer that the solutions to this system have the form

$$
\begin{aligned}
u= & F_{\mu_{1}} g_{0}+T_{\mu_{1}} F_{\lambda_{1}}\left(\operatorname{tr}_{\Gamma} T_{\mu_{1}} F_{\lambda_{1}}\right)^{-1} Q_{\mu_{1}} g_{0} \\
& +T_{\mu_{1}} \mathbf{Q}_{\lambda_{1} \mu_{1}} T_{\lambda_{1}}\left(T_{\mu_{2}} F_{\lambda_{2}}\left(\operatorname{tr}_{\Gamma} T_{\mu_{2}} F_{\lambda_{2}}\right)^{-1} Q_{\mu_{2}} g_{1}\right)+ \\
& \cdots \\
& \Pi_{j=1}^{m-1} T_{\mu_{j}} \mathbf{Q}_{\lambda_{j} \mu_{j}} T_{\lambda_{j}}\left(T_{\mu_{m}} F_{\lambda_{m}}\left(\operatorname{tr}_{\Gamma} T_{\mu_{m}} F_{\lambda_{m}}\right)^{-1} Q_{\mu_{m}} g_{m-1}\right)+ \\
& \Pi_{j=1}^{m} T_{\mu_{j}} \mathbf{Q}_{\lambda_{j} \mu_{j}} T_{\lambda_{j}}\left(h+T_{\eta} f\right)
\end{aligned}
$$


under the given boundary conditions. The smoothness of the solution $u$ is the same as in Theorem 3, i.e. of $W_{2}^{2 m+2}\left(\Omega, C l_{n}(\mathbb{R})\right)$, if we take into consideration the higher grade of smoothness. Instead of $f \in L^{2}\left(\Omega, C l_{n}(\mathbb{R})\right)$ in Theorem 3 we have $\left(h+T_{\eta} f\right) \in W_{2}^{1}\left(\Omega, C l_{n}(\mathbb{R})\right)$, so the term $\Pi_{j=1}^{m} T_{\mu_{j}} \mathbf{Q}_{\lambda_{j} \mu_{j}} T_{\lambda_{j}}\left(h+T_{\eta} f\right)$ actually is an element of $W_{2}^{2 m+1}\left(\Omega, C l_{n}(\mathbb{R})\right)$. But the other summands do not change in comparison to Theorem 3 and thus they remain in $W_{2}^{2 m+1}\left(\Omega, C l_{n}(\mathbb{R})\right)$, and so does $u$.

Remark: In case one needs a uniform regularity of all summands in the solution's representation (e.g. for controlling), the smoothness of $f$ has to be adapted by assuming $f \in W_{2}^{1}$.

\section{Explicit formulas for the solutions in balls and annular domains}

As mentioned in the beginning, the Cauchy kernel is universal for all domains. In order to evaluate the given representation formulas we however also need to compute the ortho-projectors $\mathbf{Q}_{\lambda_{j} \mu_{j}}$. These have the form $\mathbf{I}-\mathbf{P}_{\lambda_{j} \mu_{j}}$ where $\mathbf{P}_{\lambda_{j} \mu_{j}}$ is the orthogonal Bergman projection given by

$$
\begin{aligned}
& \mathbf{P}_{\lambda_{j} \mu_{j}}: L^{2}\left(\Omega, C l_{n}(\mathbb{R})\right) \rightarrow L^{2}\left(\Omega, C l_{n}(\mathbb{R})\right) \cap K \operatorname{Ker}\left(\mathbf{D}-\lambda_{j}\right)\left(\mathbf{D}-\mu_{j}\right) \\
& {\left[\mathbf{P}_{\lambda_{j} \mu_{j}} F\right](\mathbf{x}):=\int_{\Omega} B_{\lambda_{j}, \mu_{j}}(\mathbf{x}, \mathbf{y}) F(\mathbf{y}) d V(\mathbf{y}),}
\end{aligned}
$$

where we use the abbreviation $B_{\lambda_{j}, \mu_{j}}:=B_{\left(\mathbf{D}-\lambda_{j}\right)\left(\mathbf{D}-\mu_{j}\right)}$. In the particular cases where $\Omega$ is an annulus of radii $0 \leq r<R+\infty$ (where the case $r=0$ corresponds to the case dealing with a ball) we can adapt our previously obtained formulas for the Bergman kernel of the annulus of the unit ball for arbitrary polynomial Dirac equations in [9] to the particular case that we need in the representation formulas in the previous section. Let $j \in\{1, \ldots, m\}$. To proceed in this direction we first introduce the following functions:

$$
\begin{aligned}
& f_{q, \lambda_{j}}(\mathbf{x})=\|\mathbf{x}\|^{1-q-n / 2}\left(J_{q+n / 2-1}\left(\lambda_{j}\|\mathbf{x}\|\right)-\frac{\mathbf{x}}{\|\mathbf{x}\|} J_{q+n / 2}\left(\lambda_{j}\|\mathbf{x}\|\right)\right) \\
& g_{q, \lambda_{j}}(\mathbf{x})=\|\mathbf{x}\|^{1-q-n / 2}\left(Y_{q+n / 2-1}\left(\lambda_{j}\|\mathbf{x}\|\right)-\frac{\mathbf{x}}{\|\mathbf{x}\|} Y_{q+n / 2}\left(\lambda_{j}\|\mathbf{x}\|\right)\right) \quad q=0,1,2, \ldots
\end{aligned}
$$

In polar coordinates, these have the form

$$
\begin{aligned}
& f_{q, \lambda_{j}}(t \omega)=t^{1-q-n / 2}\left(J_{q+n / 2-1}\left(\lambda_{j} t\right)-\omega J_{q+n / 2}\left(\lambda_{j} t\right)\right) \\
& g_{q, \lambda_{j}}(t \omega)=t^{1-q-n / 2}\left(Y_{q+n / 2-1}\left(\lambda_{j} t\right)-\omega Y_{q+n / 2}\left(\lambda_{j} t\right)\right) \quad q=0,1,2, \ldots
\end{aligned}
$$

where $\omega \in S^{n}=\left\{\mathbf{x} \in \mathbb{R}^{n} \mid\|\mathbf{x}\|=1\right\}$ and $t>0$. Furthermore, we use the notation $S_{q}(\mathbf{x}, \mathbf{y})$ for the Szegö kernel for $\mathbf{D}_{\mathbf{x}}$-monogenic homogeneous polynomials 
of total degree $q$ in the $n$-dimensional unit ball $B(0,1)$, which equals

$$
S_{q}(\mathbf{x}, \mathbf{y})=\frac{(-1)^{q}}{A_{n}} \sum_{m=0}^{q}\left(\begin{array}{c}
n / 2-2+m \\
m
\end{array}\right)\left(\begin{array}{c}
n / 2-1+(q-m) \\
q-m
\end{array}\right)(\mathbf{x y})^{m}(\mathbf{y} \mathbf{x})^{q-m}
$$

Adapting the general formulas to the particular situation considered here, we can establish the following results:

- First case: The values $\lambda_{j}, \mu_{j}$ are both non-zero mutually distinct realbnumbers. Then the reproducing Bergman kernel for the annulus with radii $0<r<R$ has the form

$$
B_{\lambda_{j}, \mu_{j}}(\mathbf{x}, \mathbf{y})=\sum_{q=0}^{+\infty}\left(\begin{array}{c}
f_{q, \lambda_{j}} \\
f_{q, \mu_{j}} \\
g_{q, \lambda_{j}} \\
g_{q, \mu_{j}}
\end{array}\right)^{t}(\mathbf{x}) S_{q}(\mathbf{x}, \mathbf{y})\left[\mathcal{M}_{\lambda_{j}, \mu_{j}}\right]^{-1}\left(\begin{array}{c}
\bar{f}_{q, \lambda_{j}} \\
\bar{f}_{q, \mu_{j}} \\
\bar{g}_{q, \lambda_{j}} \\
\bar{g}_{q, \mu_{j}}
\end{array}\right)(\mathbf{y}) .
$$

Here the entries of the matrix $\mathcal{M}_{\lambda_{j}, \mu_{j}}$ have the form

$$
\left[\mathcal{M}_{\lambda_{j}, \mu_{j}}\right]_{k l}=\int_{r}^{R} t^{2 q+n-1} M_{k l}(t) d t \quad 1 \leq k, l \leq 4
$$

where

$$
M=\left(\begin{array}{llll}
\operatorname{Sc}\left(\bar{f}_{q, \lambda_{j}} f_{q, \lambda_{j}}\right)(t) & \operatorname{Sc}\left(\bar{f}_{q, \lambda_{j}} f_{q, \mu_{j}}\right)(t) & \operatorname{Sc}\left(\bar{f}_{q, \lambda_{j}} g_{q, \lambda_{j}}\right)(t) & \operatorname{Sc}\left(\bar{f}_{q, \lambda_{j}} g_{q, \mu_{j}}\right)(t) \\
\operatorname{Sc}\left(\bar{f}_{q, \mu_{j}} f_{q, \lambda_{j}}\right)(t) & \operatorname{Sc}\left(\bar{f}_{q, \mu_{j}} f_{q, \mu_{j}}\right)(t) & \operatorname{Sc}\left(\bar{f}_{q, \mu_{j}} g_{q, \lambda_{j}}\right)(t) & \operatorname{Sc}\left(\bar{f}_{q, \mu_{j}} g_{q, \mu_{j}}\right)(t) \\
\operatorname{Sc}\left(\bar{g}_{q, \lambda_{j}} f_{q, \lambda_{j}}\right)(t) & \operatorname{Sc}\left(\bar{g}_{q, \lambda_{j}} f_{q, \mu_{j}}\right)(t) & \operatorname{Sc}\left(\bar{g}_{q, \lambda_{j}} g_{q, \lambda_{j}}\right)(t) & \operatorname{Sc}\left(\bar{g}_{q, \lambda_{j}} g_{q, \mu_{j}}\right)(t) \\
\operatorname{Sc}\left(\bar{g}_{q, \mu_{j}} f_{q, \lambda_{j}}\right)(t) & \operatorname{Sc}\left(\bar{g}_{q, \mu_{j}} f_{q, \mu_{j}}\right)(t) & \operatorname{Sc}\left(\bar{g}_{q, \mu_{j}} g_{q, \lambda_{j}}\right)(t) & \operatorname{Sc}\left(\bar{g}_{q, \mu_{j}} g_{q, \mu_{j}}\right)(t)
\end{array}\right)
$$

and where $M_{k l}(t)$ denotes the particular matrix element of $M$ that is located in the $k$-th row and $l$-th column. Since we only consider the scalar part, all entries of $M$ and consequently also the whole integral is independent of $\omega$. The matrix values $\left[\mathcal{M}_{\lambda_{j}, \mu_{j}}\right]_{k l}$ are therefore indeed constants.

In the limit case $r \rightarrow 0$ where we are dealing with the ball $B(0, R)$ there are no outer spherical parts $g_{q}$ in the formula for the Bergman kernel. In this case the representation formula for the Bergman kernel simplifies to

$$
B_{\lambda_{j}, \mu_{j}}(\mathbf{x}, \mathbf{y})=\sum_{q=0}^{+\infty}\left(\begin{array}{l}
f_{q, \lambda_{j}} \\
f_{q, \mu_{j}}
\end{array}\right)^{t}(\mathbf{x}) S_{q}(\mathbf{x}, \mathbf{y})\left[\mathcal{M}_{\lambda_{j}, \mu_{j}}\right]^{-1}\left(\begin{array}{l}
\bar{f}_{q, \lambda_{j}} \\
\bar{f}_{q, \mu_{j}}
\end{array}\right)(\mathbf{y}) .
$$

and $\mathcal{M}_{\lambda_{j}, \mu_{j}}$ is only a $2 x 2$ matrix of the form

$$
\mathcal{M}_{\lambda_{j}, \mu_{j}}=\left(\begin{array}{ll}
\int_{0}^{R} t^{2 q+n-1} \operatorname{Sc}\left(\bar{f}_{q, \lambda_{j}} f_{q, \lambda_{j}}\right) d t & \int_{0}^{R} t^{2 q+n-1} \operatorname{Sc}\left(\bar{f}_{q, \lambda_{j}} f_{q, \mu_{j}}\right) d t \\
\int_{0}^{R} t^{2 q+n-1} \operatorname{Sc}\left(\bar{f}_{q, \mu_{j}} f_{q, \lambda_{j}}\right) d t & \int_{0}^{R} t^{2 q+n-1} \operatorname{Sc}\left(\bar{f}_{q, \mu_{j}} f_{q, \mu_{j}}\right) d t
\end{array}\right) .
$$


- Second case: $\lambda_{j}=\mu_{j} \neq 0$ :

In the first case where the values $\lambda_{j}$ and $\mu_{j}$ were distinct, the associated functions $f_{q, \lambda_{j}}, f_{q, \mu_{j}}, g_{q, \lambda_{j}}, g_{q, \mu_{j}}$ are clearly linearly independent. In this case the expression $\frac{f_{q, \mu_{j}}-f_{q, \lambda_{j}}}{\mu_{j}-\lambda_{j}}$ is linearly independent from $f_{q, \lambda_{j}}$, too. The same is true for the functions $g_{q, \lambda_{j}}$ and $\frac{g_{q, \mu_{j}}-g_{q, \lambda_{j}}}{\mu_{j}-\lambda_{j}}$. Suppose now that $\lambda_{j}=\mu_{j}$. This case evidently results from considering the limit $\mu_{j} \rightarrow \lambda_{j}$. Then $\frac{f_{q, \mu_{j}}-f_{q, \lambda_{j}}}{\mu_{j}-\lambda_{j}}$ tend to $\frac{\partial f_{q, \lambda_{j}}}{\partial \lambda_{j}}$. Then $f_{q, \lambda_{j}}$ and $\frac{\partial f_{q, \lambda_{j}}}{\partial \lambda_{j}}$ serve as linearly independent pair of functions. Similarly, $g_{q, \lambda_{j}}$ and $\frac{\partial g_{q, \lambda_{j}}}{\partial \lambda_{j}}$ serve as linearly independent pair of functions. In the case dealing with the annulus we hence obtain for the case $\lambda_{j}=\mu_{j} \neq 0$ the following representation formula for the reproducing Bergman kernel:

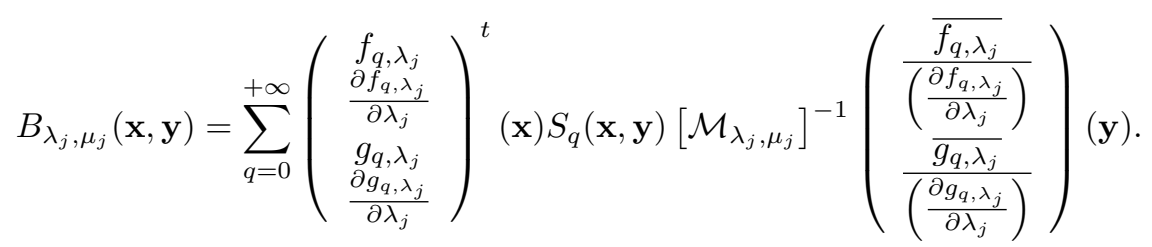

Here the matrix $\mathcal{M}_{\lambda_{j}, \mu_{j}}$ has again the form

$$
\left[\mathcal{M}_{\lambda_{j}, \mu_{j}}\right]_{k l}=\int_{r}^{R} t^{2 q+n-1} M_{k l}(t) d t \quad 1 \leq k, l \leq 4
$$

where $M_{k l}$ is given as in the previous case, but where the expressions $f_{q, \mu_{j}}$, $f_{q, \mu_{j}}, g_{q, \mu_{j}}, g_{q, \mu_{j}}$ are replaced by $\frac{\partial f_{q, \lambda_{j}}}{\partial \lambda_{j}}, \frac{\partial g_{q, \lambda_{j}}}{\partial \lambda_{j}}, \frac{\partial f_{q, \lambda_{j}}}{\partial \lambda_{j}}, \frac{\partial g_{q, \lambda_{j}}}{\partial \lambda_{j}}$, respectively. In the case dealing with the ball $B(0, R)$ we again obtained the simpler formula

$B_{\lambda_{j}, \lambda_{j}}(\mathbf{x}, \mathbf{y})=\sum_{q=0}^{+\infty}\left(\begin{array}{c}f_{q, \lambda_{j}} \\ \frac{\partial f_{q, \lambda_{j}}}{\partial \lambda_{j}}\end{array}\right)^{t}(\mathbf{x}) S_{q}(\mathbf{x}, \mathbf{y})\left[\mathcal{M}_{\lambda_{j}, \lambda_{j}}\right]^{-1}\left(\frac{\bar{f}_{q, \lambda_{j}}}{\left(\frac{\partial f_{q, \lambda_{j}}}{\partial \lambda_{j}}\right)}\right)(\mathbf{y})$.

and $\mathcal{M}_{\lambda_{j}, \lambda_{j}}$ is only a $2 \times 2$ matrix of the form

$\mathcal{M}_{\lambda_{j}, \lambda_{j}}=\left(\begin{array}{ll}\int_{0}^{R} t^{2 q+n-1} \operatorname{Sc}\left(\bar{f}_{q, \lambda_{j}} f_{q, \lambda_{j}}\right) d t & \int_{0}^{R} t^{2 q+n-1} \operatorname{Sc}\left(\bar{f}_{q, \lambda_{j}} \frac{\partial f_{q, \lambda_{j}}}{\partial \lambda_{j}}\right) d t \\ \int_{0}^{R} t^{2 q+n-1} \operatorname{Sc}\left(\overline{\frac{\partial f_{q, \lambda_{j}}}{\partial \lambda_{j}}} f_{q, \lambda_{j}}\right) d t & \int_{0}^{R} t^{2 q+n-1} \operatorname{Sc}\left(\overline{\frac{\partial f_{q, \lambda_{j}}}{\partial \lambda_{j}}} \frac{\partial f_{q, \lambda_{j}}}{\partial \lambda_{j}}\right) d t\end{array}\right)$.

- Third case: $\lambda_{j}=0, \mu_{j} \neq 0$. In view of the simplified Laurent series representation that we have according to [12] and elsewhere for monogenic functions in annular domains

$$
f(\mathbf{x})=\sum_{q=0}^{+\infty} P_{q}(\mathbf{x})+\sum_{q^{\prime}=0}^{+\infty} \frac{\mathbf{x}}{\|\mathbf{x}\|^{n+2 q^{\prime}}} P_{q^{\prime}}^{\prime}(\mathbf{x}) .
$$


Here the expressions $P_{q}$ stand for the usual homogeneous monogenic polynomials of total degree $q$. So, we can replace in this case the functions $f_{q, \lambda_{j}}$ by 1 and $g_{q, \lambda_{j}}$ by $\frac{\mathbf{x}}{\|\mathbf{x}\|^{n+2 q}}$ in the corresponding formulas for the Bergman kernel.

- Fourth case $\lambda_{j}=\mu_{j}=0$. In view of the Almansi-Fischer decomposition in this case we can replace the functions $f_{q, \lambda_{j}}$ and $f_{q, \mu_{j}}$ by $\mathbf{x}$ and $g_{q, \lambda_{j}}$ by $\frac{\mathbf{x}}{\|\mathbf{x}\|^{n+2 q}}$ and $g_{q, \mu_{j}}$ by $\frac{1}{\|\mathbf{x}\|^{n+2 q-2}}$. In this case the representation formula for the Bergman kernel for the ball and the annulus simplifies to the well known formulas for the harmonic Bergman kernel for the annulus and the ball. In the case of the ball we simply get

$$
B_{0,0}(\mathbf{x}, \mathbf{y})=\sum_{q=0}^{+\infty}\left(\begin{array}{c}
1 \\
\mathbf{x}
\end{array}\right)^{t} S_{q}(\mathbf{x}, \mathbf{y})\left[\mathcal{M}_{0,0}\right]^{-1}\left(\begin{array}{c}
1 \\
\overline{\mathbf{y}}
\end{array}\right) .
$$

where

$$
\left[\mathcal{M}_{0,0}\right]=\left(\begin{array}{ccc}
\int_{0}^{R} t^{2 q+n-1} d t & 0 \\
& 0 & \int_{0}^{R} t^{2 q+n+1} d t
\end{array}\right)
$$

which simplifies to

$$
\left[\mathcal{M}_{0,0}\right]=\left(\begin{array}{cc}
\frac{1}{2 q+n} R^{2 q+n} & 0 \\
0 & \frac{1}{2 q+n+2} R^{2 q+n+2}
\end{array}\right) .
$$

Hence, we recover the following simple formula for the harmonic Bergman kernel of the ball

$$
B_{0,0}(\mathbf{x}, \mathbf{y})=\sum_{q=0}^{+\infty}\left(\begin{array}{c}
1 \\
\mathbf{x}
\end{array}\right)^{t} S_{q}(\mathbf{x}, \mathbf{y})\left(\begin{array}{cc}
\frac{2 q+n}{R^{2 q+n}} & 0 \\
0 & \frac{2 q+n+2}{R^{2 q+n+2}}
\end{array}\right)\left(\begin{array}{c}
1 \\
-\mathbf{y}
\end{array}\right)
$$

which can directly be written as infinite sum of the well-known homogeneous Legendre polynomials, as used for instance in [10].

Final conclusion: Inserting all these formulas in the integral

$$
\left[\mathbf{Q}_{\lambda_{j} \mu_{j}} F\right](\mathbf{x})=F(\mathbf{x})-\int_{\Omega} B_{\lambda_{j}, \mu_{j}}(\mathbf{x}, \mathbf{y}) F(\mathbf{y}) d V(\mathbf{y})
$$

allow us to compute the solutions to the whole class of Dirichlet type problems posed in Section 5 explicitly.

\section{Acknowlegements}

The authors are very thankful for the fruitful discussions with Professor Dr. Wolfgang Sprößig from the Technical University of Freiberg (Germany) which lead to the successful development of this paper. 


\section{References}

[1] H. Bahmann, K. Gürlebeck, M. Shapiro and W. Sprößig: On a Modified Teodorescu Transform. Integral Transforms and Special Functions 12 No.3 (2001), pp. 213-226.

[2] S. Bernstein: Analytische Untersuchungen in unbeschränkten Gebieten mit Anwendungen auf quaternionische Operatortheorie und elliptische Randwertprobleme. PhD Thesis, Technische Universität Bergakademie Freiberg, Germany, 1993.

[3] S. Bernstein: Operator Calculus for Elliptic Boundary Value Problems in Unbounded Domains. Zeitschrift für Analysis und Ihre Anwendungen 10 No. 4 (1991), pp. 447-460.

[4] F. Brackx, and R. Delanghe: Hypercomplex function theory and Hilbert modules with reproducing kernel. Proceedings of the London Mathematical Society 37 (1978), pp. $545-576$.

[5] F. Brackx, R. Delanghe and F. Sommen: Clifford Analysis. Pitman Research Notes 76, Boston-London-Melbourne, 1982.

[6] F. Brackx, F. Sommen, and N. Van Acker: Reproducing Bergman kernels in Clifford Analysis. Complex Variables 24 (1994), pp. 191 - 204.

[7] P. Cerejeiras and U. Kähler: Elliptic boundary value problems of fluid dynamics over unbounded domains. Mathematical Methods in the Applied Sciences 23 No. 1 (2000), pp. 81-101.

[8] D. Constales, D. Grob and R.S. Kraußhar: Explicit formulas for the Green's function and the Bergman kernel for monogenic functions in annular shaped domains in $\mathbb{R}^{n+1}$. Result. Math. 58 No. 1-2 (2010), pp. 173-189.

[9] D. Constales, D. Grob and R.S. Kraußhar: Reproducing kernel functions of solutions to polynomial Dirac equations in the annulus of the unit ball in $\mathbb{R}^{n}$ and applications to boundary value problems. Journal of Mathematical Analysis and Applications 358 No. 2 (2009), pp. 281-293

[10] D. Constales, R.S. Kraußhar: Hilbert Spaces of Solutions to Polynomial Dirac equations, Fourier Transforms and Reproducing Kernel Functions for Cylindrical Domains. Zeitschrift für Analysis und Ihre Anwendungen 24 No. 3 (2005), pp. 611-636.

[11] R. Delanghe: On Hilbert modules with reproducing kernel. In: Function theoretic Methods for partial Differential Equations (Proceedings International Symposium, Darmstadt 1976, Lecture Notes in Mathematics 561 (1976), pp. $158-170$.

[12] Delanghe, R., Sommen, F. and V. Souček: Clifford Algebra and Spinor Valued Functions, Dordrecht-Boston-London: Kluwer 1992. 
[13] I. Gradshteyn, and I.M. Ryzhik: Table of Integrals, Series and Products. New York: Academic Press 1980.

[14] K. Gürlebeck, U. Kähler, J. Ryan and W. Sprößig: Clifford analysis over unbounded domains. Advances in Applied Mathematics 19 No. 2 (1997), pp. 219-239.

[15] K. Gürlebeck, K. Habetha, and W. Sprößig: Holomorphic functions in the plane and n-dimensional space. Birkhäuser Verlag, Basel, 2008

[16] K. Gürlebeck and W. Sprößig: Quaternionic analysis and elliptic boundary value problems. Basel: Birkhäuser, 1990.

[17] K. Gürlebeck and W. Sprößig: Quaternionic and Clifford Calculus for Physicists and Engineers. John Wiley \& Sons, Chichester-New York, 1997.

[18] U. Kähler: Elliptic boundary value problems in bounded and unbounded domains. In: Dirac operators in analysis, eds. J. Ryan, D. Struppa, Pitman Research Notes, Pitman Longman, 394 (1998), pp. 122-140.

[19] V.V. Kravchenko, and M. Shapiro: Quaternionic time-harmonic Maxwell operator. Journal of Physics A, Math. Gen. 28 No.17 (1995), pp. 50175031.

[20] V.V. Kravchenko, V. and M. Shapiro: Integral representations for spatial models of mathematical physics. Harlow: Addison Wesley Longman 1996.

[21] V.V. Kravchenko, and P. R. Castillo: On the kernel of the Klein-Gordon operator. Zeitschrift für Analysis und ihre Anwendungen 17 No. 2 (1998), pp. 261-265.

[22] V.V. Kravchenko: Applied quaternionic analysis. Research and Exposition in Mathematics, 28 Heldermann Verlag, Lemgo, 2003.

[23] E. Marmolejo-Olea, S. Pérez-Esteva and M. Shapiro: Modules of solutions of the Helmholtz equation arising from eigenfunctions of the Dirac operator. Bulletin of the Belgian Mathematical Society - Simon Stevin 12 No. 2 (2005), pp. 175-192.

[24] A. McIntosh and M. Mitrea: Clifford algebras and Maxwell's equations in Lipschitz domains. Mathematical Methods in the Applied Sciences 22 No.18 (1999), pp. 1599-1620.

[25] S.G. Michlin and S. Prössdorf: Singuläre Integraloperatoren. Mathematische Lehrbücher und Monographien. II. Abteilung: Mathematische Monographien, Band 52. Berlin: Akademie-Verlag, 1980.

[26] M. Mitrea: Boundary value problems and Hardy spaces associated to the Helmholtz equation in Lipschitz domains. Journal of Mathematical Analysis and Applications 202 No. 3 (1996), pp. 819-842. 
[27] J. Ryan: Cauchy-Green type formulae in Clifford analysis. Transactions of the American Mathematical Society 347 No. 4 (1995), pp. 1331-1341.

[28] M. Shapiro and N. Vasilevski: On the Bergmann kernel function in hyperholomorphic analysis. Acta Applicandae Mathematicae 46 No. 1 (1997),pp. $1-27$.

[29] F. Sommen and Zhenyuan Xu: Fundamental solutions for operators which are polynomials in the Dirac operator. In: Clifford algebras and their applications in mathematical physics, Proceedings of the 2nd Workshop, Montpellier (France) 1989, Fundamental Theories of Physics 47, Dordrecht: Kluwer 1992, pp. $313-326$.

[30] W. Sprößig: On decompositions of the Clifford Valued Hilbert Space and their Applications to Boundary Value Problems. Advances in Applied Clifford Algebras 5 No. 2 (1995), pp. 167-186.

[31] W. Sprößig: Quaternionic analysis and Maxwell's equations. Cubo Matemática Educacional 7 No. 2 (2005), pp. 57 - 67

[32] Zhenyuan $\mathrm{Xu}$ : A function theory for the operator $(D-\lambda)$. Complex Variables 16 No. 1 (1991), pp. $27-42$.

[33] Zhenyuan Xu: Helmholtz equations and boundary value problems. In: Partial differential equations with complex analysis, Pitman Res. Notes Math. Ser. 262 Longman Sci. Tech., Harlow, 1992, pp. 204-214. 